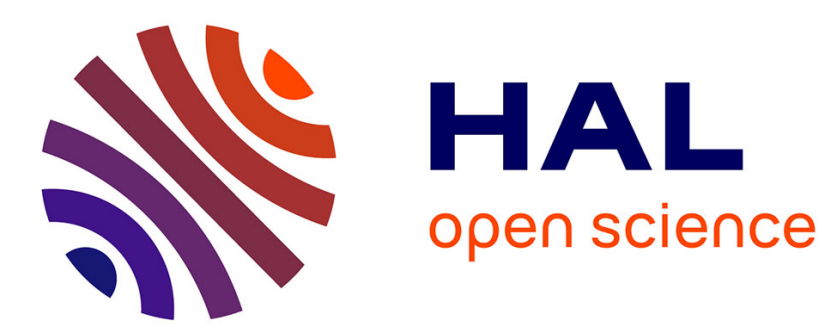

\title{
On the stability and stabilization of quaternion equilibria of rigid bodies
}

Rune Schlanbusch, Antonio Loria, P. J. Nicklasson

\section{To cite this version:}

Rune Schlanbusch, Antonio Loria, P. J. Nicklasson. On the stability and stabilization of quaternion equilibria of rigid bodies. Automatica, 2012, 48 (12), pp.3135-3141. 10.1016/j.automatica.2012.08.012 . hal-00831433

\section{HAL Id: hal-00831433 https://hal.science/hal-00831433}

Submitted on 7 Jun 2013

HAL is a multi-disciplinary open access archive for the deposit and dissemination of scientific research documents, whether they are published or not. The documents may come from teaching and research institutions in France or abroad, or from public or private research centers.
L'archive ouverte pluridisciplinaire HAL, est destinée au dépôt et à la diffusion de documents scientifiques de niveau recherche, publiés ou non, émanant des établissements d'enseignement et de recherche français ou étrangers, des laboratoires publics ou privés. 


\title{
On the Stability and Stabilization of Quaternion Equilibria of Rigid Bodies
}

\author{
Rune Schlanbusch ${ }^{\mathrm{a}}$ Antonio Loria ${ }^{\mathrm{b}}$ Per Johan Nicklasson ${ }^{\mathrm{a}}$ \\ ${ }^{a}$ Department of Scientific Computing, Electrical Engineering and Space Technology, Narvik University College, PB 385, \\ AN-8505 Narvik, Norway. E-mail: $\{$ runsch, pjn\}@hin.no \\ ${ }^{\mathrm{b}}$ CNRS, LSS-Supelec, 3 Rue Joliot Curie, Gif sur Yvette France. E-mail: loria@lss.supelec.fr
}

\begin{abstract}
We study attitude control of rigid bodies on quaternion coordinates under three mathematically different perspectives, depending on how the system dynamics are assumed to evolve. In the first case, we suppose that one equilibrium point is chosen a priori and a continuous controller is used under the assumption that the rigid body always spins in the same direction. In the second case, we relax the assumption that the sense of rotation is constant. Finally, a third scenario is considered in which hybrid (switching) control is used to choose the direction in which to spin, that is, both equilibria are continuously considered with regard to less energy consumption. It is showed that each of three scenarios must be treated in a different theoretical setting. A comparative study in simulations is also provided.
\end{abstract}

\section{Keywords:}

\section{Introduction}

The attitude of a rigid body may be described by so-called quaternions, redundant coordinates on the space $S O(3)$. Quaternions determine any point on the sphere and include one "extra" coordinate which indicates the sense of rigidbody rotations. They are redundant as the two poles of the sphere correspond to the same physical posture of the body yet, mathematically they account for two equilibria. This brings especial difficulties to the stability analysis of attitude-controlled rigid bodies.

From a practical viewpoint, certain control actions may cause the body to rotate almost a full revolution to achieve a posture which is close to the initial one, i.e., to take a longer path. From an analytical view-point the two equilibria must be considered as different hence, one may not expect to achieve "global" stability properties in closed-loop. Besides, the adjective "global" or "in the whole" pertains to the case when the states are elements of $\mathbb{R}^{n}$-see Hahn (1967). See also Loría and Panteley (2006) for precise definitions of stability and discussions.

To deal with multiple equilibria in control design there are two evident alternatives: to choose a target equilibrium before starting a maneuver, or not. If a target equilibrium is fixed before the maneuver the control design relies on the hypothesis that the sense of rotation does not change. Mathematically, this is tantamount to assuming that one of the quaternion states does not change sign. In Kristiansen et al. (2008) the authors proposes a controller which steers a spacecraft to the equilibrium point closest to the initial posture. However, the shortest-path rotation is not necessarily optimal in terms of use of input "energy" -for instance, fuel consumption in the context of spacecraft control, if initial velocities are relatively high and in direction opposite to the desired rotation. See Schlanbusch et al. (2010a) for a study of this aspect.

The freedom of not fixing the reference equilibrium $a$ priori comes at the price of the increased complexity. See for instance Casagrande et al. (2008) on control of an under-actuated non-symmetric rigid body and Mayhew et al. (2009) where the authors present two quaternion-based hybrid controllers: one is derived from an energy-based Lyapunov function which entails a switch in the rotational direction only when the rotational error is above $\pi \mathrm{rad}$ and one based on backstepping design which also considers the angular velocity errors to determine whether switching is needed.

In this paper we analyze the three following scenarios

Scenario 1.- One equilibrium is considered and is chosen a priori;

Scenario 2.- Two equilibria are considered, one of which is chosen a priori;

Scenario 3.- Two equilibria are considered, none of which is chosen a priori.

For comparison, we use in the three cases a controller that is inspired from Slotine and $\mathrm{Li}$ (1988). However, the controller that we propose is adapted to the rigid body in quaternion space hence, it is different from that in the latter reference, which applies to robot manipulators in joint space $\left(\mathbb{R}^{2 n}\right)^{1}$. In the first case, the controller is showed to guarantee asymptotic stability in the large provided that the sense

1 We remark that the choice of the controller is unimportant, i.e., the same results may be obtained for many other controllers inspired from robot control literature. 
of rotation is constant. In the second case, we add to the previous controller a switching rule which yields only one potential initial switch and we provide a proof of asymptotic stability using density functions -see Rantzer (2001). We show asymptotic stability for all initial conditions on the sphere except for a zero-measure set. In the third case we incorporate a switching law, the closed-loop system is hybrid and we use the framework of Sanfelice et al. (2007) to analyze the closed-loop system.

Simulation results are presented with the aim at showing that a proper design of the switching law may lead to performance improvement.

The rest of the paper is organized as follows. In Section 2 we describe the quaternion-based model of a rigid-body; in Section 3 we present our main results; in Section 4 we present a comparative simulations study and we conclude with some remarks in Section 5.

\section{Rigid-body model}

Attitude control consists in achieving any rigid-body orientation relative to a fixed frame, independent of that attached to the body itself. Perhaps the best manner to explain the kinematics and dynamics is to consider the attitude of a satellite relative to the Earth. We denoted the body frame as $\mathcal{F}^{b}$, and is located at the center of mass of the rigid body, and its basis vectors are aligned with the main axis of inertia and the inertia frame as $\mathcal{F}^{i}$.

\subsection{Quaternions}

We recall that the special orthogonal group of order three corresponds to the set $S O(3)$ of orthonormal rotation matrices $\mathbf{R}$

$$
S O(3)=\left\{\mathbf{R} \in \mathbb{R}^{3 \times 3}: \mathbf{R}^{\top} \mathbf{R}=\mathbf{I}, \operatorname{det}(\mathbf{R})=1\right\},
$$

where I denotes the identity matrix. A rotation matrix for a rotation $\theta$ about an arbitrary unit vector $\mathbf{k}$ may be angleaxis parameterized as in Egeland and Gravdahl (2002), i.e.,

$$
\mathbf{R}_{k, \theta}=\mathbf{I}+\mathbf{S}(\mathbf{k}) \sin (\theta)+\mathbf{S}^{2}(\mathbf{k})(1-\cos (\theta)) .
$$

Then, the coordinate transformation of a vector $\mathbf{r}$ from frame $a$ to frame $b$ is written as $\mathbf{r}^{b}=\mathbf{R}_{a}^{b} \mathbf{r}^{a}$. The rotation matrix in (1) can be expressed by an Euler parameter representation as

$$
\mathbf{R}=\mathbf{I}+2 \eta \mathbf{S}(\boldsymbol{\epsilon})+2 \mathbf{S}^{2}(\boldsymbol{\epsilon})
$$

where the matrix $\mathbf{S}(\cdot)$ is the cross product operator, i.e.,

$$
\mathbf{S}(\boldsymbol{\epsilon})=\left[\begin{array}{ccc}
0 & -\epsilon_{z} & \epsilon_{y} \\
\epsilon_{z} & 0 & -\epsilon_{x} \\
-\epsilon_{y} & \epsilon_{x} & 0
\end{array}\right], \quad \boldsymbol{\epsilon}=\left[\begin{array}{c}
\epsilon_{x} \\
\epsilon_{y} \\
\epsilon_{z}
\end{array}\right]
$$

Quaternions are used to parameterize elements of $S O(3)$. The unit quaternion is defined as $\mathbf{q}=\left[\eta, \boldsymbol{\epsilon}^{\top}\right]^{\top} \in S^{3}=$ $\left\{\mathbf{x} \in \mathbb{R}^{4}: \mathbf{x}^{\top} \mathbf{x}=1\right\}$, where $\eta=\cos (\theta / 2) \in \mathbb{R}$ is 'the scalar part' and $\boldsymbol{\epsilon}=\mathbf{k} \sin (\theta / 2) \in \mathbb{R}^{3}$ is 'the vector part'. The set $S^{3}$ forms a group with quaternion multiplication, which is distributive and associative, but not commutative.
The inverse rotation of a given attitude is performed via the inverse conjugated $\overline{\mathbf{q}}=\left[\eta,-\boldsymbol{\epsilon}^{\top}\right]^{\top}$.

The difference between two postures is given by the quaternion product,

$$
\mathbf{q}_{1} \otimes \mathbf{q}_{2}=\left[\begin{array}{c}
\eta_{1} \eta_{2}-\boldsymbol{\epsilon}_{1}^{\top} \boldsymbol{\epsilon}_{2} \\
\eta_{1} \boldsymbol{\epsilon}_{2}+\eta_{2} \boldsymbol{\epsilon}_{1}+\mathbf{S}\left(\boldsymbol{\epsilon}_{1}\right) \boldsymbol{\epsilon}_{2}
\end{array}\right]
$$

We stress that the quaternion representation is redundant. Notice that $\mathbf{q}$ and $\mathbf{- q}$ represent the same physical attitude however, the two postures differ mathematically by a $2 \pi$ rotation about an arbitrary axis. As a consequence, the mathematical model has two equilibria and this must be considered when studying stability.

\subsection{Kinematics and Dynamics}

The time derivative of the rotation matrix is

$$
\dot{\mathbf{R}}_{b}^{a}=\mathbf{S}\left(\boldsymbol{\omega}_{a, b}^{a}\right) \mathbf{R}_{b}^{a}=\mathbf{R}_{b}^{a} \mathbf{S}\left(\boldsymbol{\omega}_{a, b}^{b}\right)
$$

where $\boldsymbol{\omega}_{a, b}^{a} \in \mathbb{R}^{3}$ is the angular velocity of a frame $\mathcal{F}^{b}$ relative to a frame $\mathcal{F}^{a}$, expressed in frame $\mathcal{F}^{a}$. Correspondingly, the kinematic equation is

$$
\dot{\mathbf{q}}=\mathbf{T}(\mathbf{q}) \boldsymbol{\omega}, \quad \mathbf{T}(\mathbf{q})=\frac{1}{2}\left[\begin{array}{c}
-\boldsymbol{\epsilon}^{T} \\
\eta \mathbf{I}+\mathbf{S}(\boldsymbol{\epsilon})
\end{array}\right] \in \mathbb{R}^{4 \times 3}
$$

The rigid body dynamics is derived from Euler's moment equation, which describes the relation between applied torque and angular momentum on the rigid body, i.e.,

$$
\boldsymbol{J} \dot{\boldsymbol{\omega}}=-\mathbf{S}(\boldsymbol{\omega}) \mathbf{J} \boldsymbol{\omega}+\boldsymbol{\tau}
$$

where $\boldsymbol{\omega}=\boldsymbol{\omega}_{i, b}^{b}$ is the angular velocity of the body frame $\mathcal{F}^{b}$ relative to an inertia frame $\mathcal{F}^{i}$, expressed in the body frame; $\tau \in \mathbb{R}^{3}$ is the total torque working on the body frame ${ }^{2}$, and $\mathbf{J} \in \mathbb{R}^{3 \times 3}=\operatorname{diag}\left\{J_{x}, J_{y}, J_{z}\right\}$ is the inertia matrix.

\section{Attitude control on quaternion coordinates}

The attitude control problem consists in making the actual attitude converge towards a given reference attitude $\mathbf{q}_{d}$ satisfying the kinematic equation

$$
\dot{\mathbf{q}}_{d}=\mathbf{T}\left(\mathbf{q}_{d}\right) \boldsymbol{\omega}_{d}
$$

Assumption 1 a) The desired attitude $\mathbf{q}_{d}$, the desired angular velocity $\boldsymbol{\omega}_{d}$ and the desired angular acceleration $\dot{\boldsymbol{\omega}}_{d}$ are all bounded functions; b) the desired reference is such that the quaternion errors satisfy the quaternion constraint $\tilde{\boldsymbol{\epsilon}}^{\top} \tilde{\boldsymbol{\epsilon}}=1-\tilde{\eta}^{2}$.

The quaternion error is given by $\tilde{\mathbf{q}}=\overline{\mathbf{q}}_{d} \otimes \mathbf{q}$ and yields $\tilde{\mathbf{q}}=$ $\left[\tilde{\eta}, \tilde{\boldsymbol{\epsilon}}^{\dagger}\right]^{\top}$ with $\tilde{\eta} \in[-1,1]$, by definition. The control goal is to steer $\tilde{\boldsymbol{\epsilon}}(t)$ to zero under Assumption 1. Correspondingly, in view of the quaternion constraint, $\tilde{\eta}$ must converge either to +1 or to -1 .

2 Typically, in the context of attitude control of spacecraft, $\tau$ contains the control inputs and external disturbances. The latter are not considered in this paper. 
Now, the error kinematic equation is given by

$$
\dot{\tilde{\mathbf{q}}}=\mathbf{T}(\tilde{\mathbf{q}}) \boldsymbol{e}_{\omega}
$$

where $\mathbf{e}_{\omega}=\boldsymbol{\omega}-\boldsymbol{\omega}_{d}$. We remark that due to the redundancy of the quaternion coordinates (3) has two equilibria, which we represent by $\left(\tilde{\mathbf{q}}_{+}, \mathbf{e}_{\omega}\right)=([1 \mathbf{0}], \mathbf{0})$ and $\left(\tilde{\mathbf{q}}_{-}, \mathbf{e}_{\omega}\right)=$ $([-1 \mathbf{0}], \mathbf{0})$ where $\mathbf{0}=[0 \cdots 0]^{\top}$ is of appropriate dimensions. For the purpose of analysis we translate the problem of stabilizing an equilibrium to that of stabilizing the origin. For this, we define the attitude error vector $\mathbf{e}_{q+}=$ $\left[1-\tilde{\eta}, \tilde{\boldsymbol{\epsilon}}^{\top}\right]^{\top}$ for the "positive" equilibrium and use $\mathbf{e}_{q-}=$ $\left[1+\tilde{\eta}, \tilde{\boldsymbol{\epsilon}}^{\top}\right]^{\top}$ for the "negative" equilibrium. The kinematic relation can then be expressed as

$$
\dot{\mathbf{e}}_{q \pm}=\mathbf{T}_{e}\left(\mathbf{e}_{q \pm}\right) \mathbf{e}_{\omega}, \text { where } \mathbf{T}_{e}\left(\mathbf{e}_{q \pm}\right)=\frac{1}{2}\left[\begin{array}{c} 
\pm \tilde{\boldsymbol{\epsilon}}^{\top} \\
\tilde{\eta} \mathbf{I}+S(\tilde{\boldsymbol{\epsilon}})
\end{array}\right]
$$

In the first two scenarios described below, the control strategy relies on choosing a target equilibrium before the manoeuvre. In the first case, we assume that the body's sense of rotation is constant hence the state space is restricted by the constraint $\tilde{\eta} \geq 0$ or $\tilde{\eta} \leq 0$; in the second case we relax such assumption. In both scenarios the control action is generated by

$$
\begin{aligned}
\boldsymbol{\tau} & =\mathbf{J} \dot{\boldsymbol{\omega}}_{r}-\mathbf{S}(\mathbf{J} \boldsymbol{\omega}) \boldsymbol{\omega}_{r}-k_{q} \mathbf{T}_{e}^{\top} \mathbf{e}_{q}-k_{\omega}\left(\boldsymbol{\omega}-\boldsymbol{\omega}_{r}\right), \\
\boldsymbol{\omega}_{r} & =\boldsymbol{\omega}_{d}-\gamma \mathbf{T}_{e}^{\top} \mathbf{e}_{q},
\end{aligned}
$$

where $k_{q}>0, k_{\omega}>0$ and $\gamma>0$ and $\mathbf{T}_{e}$ is defined by (4), that is, it depends on the choice of the target equilibrium, made before the manoeuvre.

Define $\mathbf{s}=\boldsymbol{\omega}-\boldsymbol{\omega}_{r}$. Then, the closed-loop system is obtained by differentiating the latter, using (5a) in (2) and using (5b) with $\mathbf{s}=\boldsymbol{\omega}-\boldsymbol{\omega}_{r}$ in (4), that is,

$$
\left[\begin{array}{c}
\dot{\mathbf{e}}_{q} \\
\dot{\mathbf{s}}
\end{array}\right]=\left[\begin{array}{c}
\mathbf{T}_{e} \mathbf{s}-\mathbf{T}_{e} \gamma \mathbf{T}_{e}^{\top} \mathbf{e}_{q} \\
\mathbf{J}^{-1}\left\{\left[\mathbf{S}(\mathbf{J} \boldsymbol{\omega}(t))-k_{\omega} \mathbf{I}\right] \mathbf{s}-k_{q} \mathbf{T}_{e}^{\top} \mathbf{e}_{q}\right\}
\end{array}\right]
$$

Note that independently of whether $\mathbf{e}_{q}=\mathbf{e}_{q+}$ or $\mathbf{e}_{q}=\mathbf{e}_{q-}$ the right hand-side of (6) equals $[\mathbf{0}, \mathbf{0}]^{\top}$ if $\mathbf{s}=\mathbf{0}$ and $\tilde{\boldsymbol{\epsilon}}=\mathbf{0}$ where the latter holds if and only if $\tilde{\mathbf{q}}=[1,0,0,0]^{\top}$ or $\tilde{\mathbf{q}}=[-1,0,0,0]^{\top}$.

In both scenarios we show that the following stability property is ensured ${ }^{3}$ with respect to different sets.

Definition 2 (Asymptotic stability in the large) Let $\Gamma \subset \mathbb{R}^{n}$ be given. The trivial solution $x=0$ of $\dot{x}=f(t, x)$ is called asymptotically stable in the large with respect to $\Gamma$ if it is stable in the sense of Lyapunov and every other solution $x\left(t, t_{\circ}, x_{\circ}\right) \rightarrow 0$ as $t \rightarrow \infty$ for any initial states $x_{\circ} \in \Gamma$ and for any initial times $t_{\circ} \in \mathbb{R}_{\geq 0}$. The origin is uniformly asymptotically stable if it is uniformly stable and convergence is uniform in the initial times and in compact sets of the initial states.

\footnotetext{
3 The definition is paraphrased from (Furasov, 1977, p. 29) for the purposes of this paper.
}

\subsection{Scenario 1: the sense of rotation does not change}

We consider that the state space of (6) corresponds to either $\Gamma_{+}:=\left\{\mathbf{e}_{q+}: \tilde{\mathbf{q}} \in S^{3}, \tilde{\eta} \in[0,1]\right\} \times \mathbb{R}^{3}$ or $\Gamma_{-}:=\left\{\mathbf{e}_{q-}:\right.$ $\left.\tilde{\mathbf{q}} \in S^{3}, \tilde{\eta} \in[-1,0]\right\} \times \mathbb{R}^{3}$, depending on which target equilibrium is chosen.

Proposition 3 Let Assumption 1 hold and let $\Gamma_{+}^{\circ} \subset$ $\Gamma_{+}$. Assume that all trajectories with initial conditions $\left(t_{\circ}, \mathbf{e}_{q+}\left(t_{\circ}\right), \mathbf{e}_{\omega}\left(t_{\circ}\right)\right) \in \mathbb{R}_{\geq 0} \times \Gamma_{+}^{\circ} \operatorname{satisfy} \operatorname{sgn}(\tilde{\eta}(t))=$ $\operatorname{sgn}\left(\tilde{\eta}\left(t_{\circ}\right)\right) \geq 0$ for all $t \geq t_{\circ}\left[\right.$ mutatis mutandis for $\left.\Gamma_{-}^{\circ}\right]$. Then, the equilibrium point $\left(\mathbf{e}_{q+}, \mathbf{e}_{\omega}\right)=(\mathbf{0}, \mathbf{0})$ [respectively, the equilibrium $\left.\left(\mathbf{e}_{q-}, \mathbf{e}_{\omega}\right)=(\mathbf{0}, \mathbf{0})\right]$ of system (6) is uniformly asymptotically stable in the large with respect to $\Gamma_{+}^{\circ}$ [ respectively, with respect to $\Gamma_{-}^{\circ}$ ].

Remark 3.1 An estimate of $\Gamma_{+}^{\circ}$, respectively of $\Gamma_{-}^{\circ}$, may be obtained using Lyapunov theory and restricting the size of initial velocity and attitude errors -cf. Caccavale and Villani (1999).

Proof: Define $\mathbf{x}=\left[\mathbf{s}^{\top}, \mathbf{e}_{q}^{\top}\right]^{\top}$. Without loss of generality, let the target equilibrium point be $\left(\mathbf{e}_{q+}, \mathbf{e}_{\omega}\right)=(\mathbf{0}, \mathbf{0})$. Then, the closed-loop equations are given by (6). By Assumption the trajectories that originate in $\Gamma_{+}^{\circ} \operatorname{satisfy} \operatorname{sgn}(\tilde{\eta}(t))=$ $\operatorname{sgn}\left(\tilde{\eta}\left(t_{\circ}\right)\right) \geq 0$ for all $t \geq t_{\circ}$ hence, in view of Assumption 1 (the quaternion constraint) $\mathbf{x}(t) \in \Gamma_{+}$for all $t \geq t_{\circ}$. Consider the radially unbounded, positive definite Lyapunov function candidate $V: \Gamma_{+} \rightarrow \mathbb{R}_{\geq 0}$,

$$
V(\mathbf{x})=\frac{1}{2} \mathbf{s}^{\top} \mathbf{J} \mathbf{s}+\frac{1}{2} \mathbf{e}_{q}^{\top} k_{q} \mathbf{e}_{q}
$$

which satisfies

$$
\dot{V}(\mathbf{x})=\mathbf{s}^{\top} \mathbf{J} \dot{\mathbf{s}}+\mathbf{e}_{q}^{\top} k_{q} \dot{\mathbf{e}}_{q} .
$$

Using (6) and the skew-symmetry of $\mathbf{S}$ we obtain

$$
\dot{V}(\mathbf{x})=-\mathbf{s}^{\top} k_{\omega} \mathbf{s}-\mathbf{e}_{q}^{\top} \mathbf{T}_{e} \gamma k_{q} \mathbf{T}_{e}^{\top} \mathbf{e}_{q}
$$

which under the quaternion constraint, implies that

$$
\dot{V}(\mathbf{x}) \leq-\mathbf{s}^{\top} k_{\omega} \mathbf{s}-\mathbf{e}_{q}^{\top} \frac{\gamma k_{q}}{8} \mathbf{e}_{q} ;
$$

to see this notice that $\mathbf{T}_{e}^{\top} \mathbf{e}_{q}=(1 / 2) \tilde{\boldsymbol{\epsilon}}$ hence,

$$
\mathbf{e}_{q}^{\top} \mathbf{T}_{e} \mathbf{T}_{e}^{\top} \mathbf{e}_{q}=\frac{1}{4} \tilde{\boldsymbol{\epsilon}}^{\top} \tilde{\boldsymbol{\epsilon}}
$$

Now, assume that

$$
\frac{1}{4} \tilde{\boldsymbol{\epsilon}}^{\top} \tilde{\boldsymbol{\epsilon}}<\frac{1}{8}\left[(1-\tilde{\eta})^{2}+\tilde{\boldsymbol{\epsilon}}^{\top} \tilde{\boldsymbol{\epsilon}}\right]
$$

which is equivalent to

$$
(1-\tilde{\eta})^{2}>\tilde{\boldsymbol{\epsilon}}^{\top} \tilde{\boldsymbol{\epsilon}}
$$

Then, in view of the unit quaternion constraint $\tilde{\boldsymbol{\epsilon}}^{\top} \tilde{\boldsymbol{\epsilon}}=1-$ $\tilde{\eta}^{2}$, inequality (12) holds if and only if $\tilde{\eta}^{2}>\tilde{\eta}$ which is impossible since $|\tilde{\eta}| \in[0,1]$. We conclude that (11) does not hold hence, from (10) and $\mathbf{e}_{q}^{\top} \mathbf{e}_{q}=\left[(1-\tilde{\eta})^{2}+\tilde{\boldsymbol{\epsilon}}^{\top} \tilde{\boldsymbol{\epsilon}}\right]$, we obtain

$$
\mathbf{e}_{q}^{\top} \mathbf{T}_{e} \mathbf{T}_{e}^{\top} \mathbf{e}_{q} \geq \frac{1}{8} \mathbf{e}_{q}^{\top} \mathbf{e}_{q}
$$


which together with (8), implies (9). The proof is completed invoking standard Lyapunov theory arguments. Let $j_{M} \geq\|\mathbf{J}\| \geq j_{m}, c_{1}:=0.5 \min \left\{j_{m}, k_{q}\right\}, c_{2}:=\max \left\{j_{M}, k_{q}\right\}$ and $c_{3}:=\min \left\{k_{\omega}, \gamma k_{q} / 8\right\}$. Then, from (7) we have $c_{2}\left[\|\mathbf{s}\|^{2}+\left\|\mathbf{e}_{q}\right\|^{2}\right] \geq V(\mathbf{x}) \geq c_{1}\left[\|\mathbf{s}\|^{2}+\left\|\mathbf{e}_{q}\right\|^{2}\right]$ and, together with (9) we obtain $\dot{V}(\mathbf{x}) \leq-\left(c_{3} / c_{2}\right) V(\mathbf{x})$. All these inequalities hold for all $x \in \Gamma_{+}$. The result follows recalling that $\mathbf{e}_{\omega}=\gamma \mathbf{T}_{e}^{\top} \mathbf{e}_{q}-\mathbf{s}$.

The proof for the negative equilibrium follows $m u$ tatis mutandis, replacing $\Gamma_{+}$with $\Gamma_{-}$and noting that $\mathbf{T}_{e}\left(\mathbf{e}_{q-}\right)^{\top} \mathbf{e}_{q}=-(1 / 2) \tilde{\boldsymbol{\epsilon}}$ so the previous computations remain valid.

\subsection{Scenario 2: two equilibria}

We relax the assumption that the sign of $\tilde{\eta}(t)$ is constant for all $t$ and consider that the state space of the closedloop system is $\Gamma=\Gamma_{+} \cup \Gamma_{-}$. In this space, the closed-loop system possesses two equilibria: the positive equilibrium $\left(\mathbf{e}_{q+}, \mathbf{e}_{\omega}\right)=(\mathbf{0}, \mathbf{0})$ and the negative equilibrium $\left(\mathbf{e}_{q-}, \mathbf{e}_{\omega}\right)=$ $(\mathbf{0 , 0})$. As in Rantzer (2001); Angeli (2004, 2001), we use a notion of stability for all initial states except for a zeromeasure set. For systems with state space $\mathbb{R}^{n}$, we speak of almost global asymptotic stability -see Rantzer (2001) if the origin is asymptotically stable for all all initial states in $\mathbb{R}^{n}$ except for a set of measure zero. If not because the system's state space is a subset of $\mathbb{R}^{6}$ (hence, we cannot use the qualifier 'global') this is the property that we establish below, relying on a refinement of the main theorem in Rantzer (2001) for non-autonomous systems. Define

$$
\begin{aligned}
\nabla \cdot f & :=\frac{\partial f_{1}}{\partial x_{1}}+\cdots+\frac{\partial f_{n}}{\partial x_{n}}, \quad f: \mathbb{R} \times \mathbb{R}^{n} \rightarrow \mathbb{R}^{n}, \\
\nabla V & :=\left[\frac{\partial V}{\partial x_{1}} \cdots \frac{\partial V}{\partial x_{n}}\right], \quad V: \mathbb{R}^{n} \rightarrow \mathbb{R} .
\end{aligned}
$$

Theorem 4 Consider the system $\dot{x}=f(t, x)$ such that $f(t, 0)=0$ for all $t$ and 0 is a locally stable equilibrium point. Let $\rho: \mathbb{R}^{n} \backslash\{0\} \rightarrow \mathbb{R}_{\geq 0}$ be once continuously differentiable and satisfy

$$
\frac{\partial}{\partial t} \rho(t, x)+[\nabla \cdot(f \rho)](t, x)>0 \quad \forall t \geq 0, \text { a.a. } x \in \mathbb{R}^{n} .
$$

Moreover, assume that $\rho(t, x)$ is integrable uniformly in $t$ over $\{\|x\|>\varepsilon\}$ for every $\varepsilon>0$. Then, for every initial time $t_{\circ}$ the set of points that are not asymptotically attracted by the origin has zero Lebesgue measure.

\section{Remark 3.2}

- The proof of Theorem 4 follows along the lines of the proof of Proposition 2.2. of Monzón (2006); actually, the enunciate of Theorem 4 corresponds to that of Proposition 2.2. of Monzón (2006) except that in the latter reference the author assumes that " $\rho(t, x)$ is integrable for fixed $t$ ".

- Stability is an assumption of Theorem 4 hence, an implicit statement which follows directly from its conclusion is that the origin is almost globally asymptotically stable, in the sense defined in Rantzer (2001).

Proposition 5 Let Assumption 1 hold. The equilibrium point $\left(\mathbf{e}_{q+}, \mathbf{e}_{\omega}\right)=(\mathbf{0}, \mathbf{0})$ [respectively, the equilibrium $\left.\left(\mathbf{e}_{q-}, \mathbf{e}_{\omega}\right)=(\mathbf{0}, \mathbf{0})\right]$ of the closed-loop system (6) is asymptotically stable in the large with respect to $\Gamma$, taken away a set of measure zero which includes the dual equilibrium $\left\{\left(\mathbf{e}_{q-}, \mathbf{e}_{\omega}\right)=(\mathbf{0}, \mathbf{0})\right\}$ [respectively, with respect to $\Gamma$, taken away a set of measure zero including the dual equilibrium $\left.\left\{\left(\mathbf{e}_{q+}, \mathbf{e}_{\omega}\right)=(\mathbf{0}, \mathbf{0})\right\}\right]$.

In other words, the equilibrium point $\left(\mathbf{e}_{q+}, \mathbf{e}_{\omega}\right)=(\mathbf{0}, \mathbf{0})$ [respectively, the equilibrium $\left.\left(\mathbf{e}_{q-}, \mathbf{e}_{\omega}\right)=(\mathbf{0}, \mathbf{0})\right]$ is stable and attracts almost all trajectories in $\Gamma$.

Proof: Firstly, we observe that local stability of either equilibrium follows from the proof of Proposition 3; the proof of convergence from all initial states in $\Gamma$ taken away a zeromeasure set, relies on Theorem 4 . Without loss of generality let $\mathbf{e}_{q}=\mathbf{e}_{q+}$ and define $\mathbf{x}=\left[\mathbf{e}_{q}^{\top}, \mathbf{s}^{\top}\right]^{\top}$. We apply Theorem 4 with $x=\mathrm{x}$ hence, let $f$ denote the expression on the right-hand side of (6) with $\mathbf{T}_{e}=\mathbf{T}_{e}\left(\mathbf{e}_{q+}\right)$ and consider the density function $\rho: \Gamma \backslash\{\mathbf{x}=\mathbf{0}\} \rightarrow \mathbb{R}_{\geq 0}$,

$$
\rho(\mathbf{x})=\left[\frac{1}{2}\left(\mathbf{e}_{q}^{\top} k_{q} \mathbf{e}_{q}+\mathbf{s}^{\top} \mathbf{J} \mathbf{s}\right)\right]^{-\alpha}, \quad \alpha>2 .
$$

That is, we have $\rho(\mathbf{x})=V(\mathbf{x})^{-\alpha}$ where $V: \Gamma \rightarrow \mathbb{R}_{\geq 0}$ is defined as in (7). Since $V(\mathbf{x})=\mathcal{O}\left(\|\mathbf{x}\|^{2}\right)$ and $\rho$ is independent of $t$ the integrability condition on $\rho$ holds provided that $\alpha>2$. It is left to show that

$$
[\nabla \cdot(f \rho)](t, \mathbf{x})>0 \quad \forall t \geq 0, \text { a.a. } \mathbf{x} \in \Gamma .
$$

As in the first steps of the proof of (Rantzer, 2001, Proposition 1), we have ${ }^{4}$

$$
\begin{aligned}
\nabla \cdot(f \rho) & =\nabla \rho \cdot f+(\nabla \cdot f) \rho \\
& =(\nabla \cdot f) V^{-\alpha}-\alpha V^{-(\alpha+1)} \nabla V \cdot f \\
& =V^{-(\alpha+1)}[(\nabla \cdot f) V-\alpha \nabla V \cdot f] .
\end{aligned}
$$

The right-hand side of the previous equality is positive if and only if so is the sum in brackets. Let us show this. On one hand we have

$$
\nabla V \cdot f=-\mathbf{s}^{\top} k_{\omega} \mathbf{s}-\mathbf{e}_{q}^{\top} \mathbf{T}_{e} \gamma k_{q} \mathbf{T}_{e}^{\top} \mathbf{e}_{q}
$$

and on the other ${ }^{5}$,

$$
\begin{gathered}
{[\nabla \cdot f](t, \mathbf{x})=\nabla\left(\mathbf{e}_{q}\right) \cdot \dot{\mathbf{e}}_{q}+\nabla(\mathbf{s}) \cdot \dot{\mathbf{s}}} \\
\nabla\left(\mathbf{e}_{q}\right) \cdot \dot{\mathbf{e}}_{q}=\nabla\left(\mathbf{e}_{q}\right) \cdot\left(\mathbf{T}_{e} \mathbf{s}-\gamma \mathbf{T}_{e} \mathbf{T}_{e}^{\top} \mathbf{e}_{q}\right)=-\frac{3 \gamma \tilde{\eta}}{4} \\
\nabla(\mathbf{s}) \cdot \dot{\mathbf{s}}=\nabla(\mathbf{s}) \cdot\left(\mathbf{J}^{-1}\left\{\left[\mathbf{S}(\mathbf{J} \boldsymbol{\omega}(t))-k_{\omega} \mathbf{I}\right] \mathbf{s}-k_{q} \mathbf{T}_{e}^{\top} \mathbf{e}_{q}\right\}\right) .
\end{gathered}
$$

Then, using $\boldsymbol{\omega}(t)=\mathbf{s}+\boldsymbol{\omega}_{r}(t)$ we obtain

$$
\nabla(\mathbf{s}) \cdot \dot{\mathbf{s}}=\nabla \cdot\left(\mathbf{J}^{-1}\left\{\left[\mathbf{S}(\mathbf{J} \mathbf{s})+\mathbf{S}\left(\mathbf{J} \boldsymbol{\omega}_{r}(t)\right)-k_{\omega} \mathbf{I}\right] \mathbf{s}-k_{q} \mathbf{T}_{e}^{\top} \mathbf{e}_{q}\right\}\right)
$$

and since

$$
\begin{gathered}
\nabla(\mathbf{s}) \cdot \mathbf{S}(\mathbf{J} \mathbf{s}) \mathbf{s}=0 \\
\nabla(\mathbf{s}) \cdot \mathbf{S}\left(\mathbf{J} \boldsymbol{\omega}_{r}(t)\right) \mathbf{s}=0
\end{gathered}
$$

4 We drop the arguments $t, \mathbf{x}$ for simplicity in the notation. 5 With an abuse of notation we split up the gradient and the vector with respect to which the function is differentiated hence, we write $\nabla(x) \cdot f(x, y)=\frac{\partial f(x, y)}{\partial x}$. 
for any $t$, we see that

$$
\nabla(\mathbf{s}) \cdot \dot{\mathbf{s}}=-3 c k_{\omega}
$$

where $c:=\left(1 / J_{x}+1 / J_{y}+1 / J_{z}\right)$. Therefore,

$$
[\nabla \cdot f](t, \mathbf{x})=-3\left(\frac{\gamma \tilde{\eta}}{4}+c k_{\omega}\right)
$$

Using (14), (15) and (7) we see that

$$
[(\nabla \cdot f) V-\alpha \nabla V \cdot f]>0
$$

if and only if

$$
\begin{aligned}
-\frac{3}{2}\left(\frac{\gamma \tilde{\eta}}{4}+c k_{\omega}\right)\left[\mathbf{s}^{\top} \mathbf{J} \mathbf{s}+\frac{1}{2} \mathbf{e}_{q}^{\top} k_{q} \mathbf{e}_{q}\right] & \\
+ & \alpha\left[\mathbf{s}^{\top} k_{\omega} \mathbf{s}+\mathbf{e}_{q}^{\top} \mathbf{T}_{e} \gamma k_{q} \mathbf{T}_{e}^{\top} \mathbf{e}_{q}\right]>0 .
\end{aligned}
$$

In view of (13) we see that the previous inequality holds for sufficiently large values of $\alpha$ and provided that $\mathbf{x} \neq 0$ (which correspond to the two equilibria). According to Theorem 4 all points in $\Gamma$ except for a set of measure zero, generate via (6) with $\mathbf{T}_{e}=\mathbf{T}_{e}\left(\mathbf{e}_{q+}\right)$, solutions that converge asymptotically to $\left(\mathbf{e}_{q+}, \mathbf{e}_{\omega}\right)=(\mathbf{0}, \mathbf{0})$. It is evident that the dual equilibrium $\left(\mathbf{e}_{q-}, \mathbf{e}_{\omega}\right)=(\mathbf{0}, \mathbf{0})$ also generates trajectories a trajectory which does not converge to $\left(\mathbf{e}_{q+}, \mathbf{e}_{\omega}\right)=(\mathbf{0}, \mathbf{0})$.

A similar analysis with the pertinent modifications, may be established for the dual equilibrium $\mathbf{e}_{q}=\mathbf{e}_{q-}$.

\subsection{Scenario 3: Hybrid control}

We assume now that the controller is hybrid. In particular, we introduce a switching law which determines the reference equilibrium online. That is, the latter is not fixed before the maneuver. For the sake of comparison, the continuous-time component of the controller corresponds to the algorithm previously used. Hysteresis is introduced to avoid chattering.

For the purpose of analysis, we use the setting of Sanfelice et al. (2007); Goebel et al. (2009). According with this framework hybrid systems are described by a continuoustime dynamics defined by a "flow map" and discrete-time dynamics, defined by a "jump map". In addition, we are equipped of a "flow set" and a "jump set". That is,

$$
\mathcal{H}:\left\{\begin{array}{l}
x \in C \Longrightarrow \dot{x}=F(x) \\
x \in D \Longrightarrow x^{+}=G(x)
\end{array}\right.
$$

where $x^{+}$is the state value 'immediately' after a jump.

After Sanfelice et al. (2007); Goebel et al. (2009) solutions to the hybrid system are defined as maps from a hybrid time domain, subset of $\mathbb{R}_{\geq 0} \times \mathbb{N}$, into an Euclidean space. Roughly, the hybrid time domain denoted "dom $x$ ", consists in an ordered sequence of continuous-time intervals $\left[t_{j}, t_{j+1}\right)$ or $\left[t_{j}, t_{j+1}\right]$ and discrete instants $\{j\}$. During flows (if $x(t, j) \in C$ ) the solution is a locally absolutely continuous function that satisfies $\dot{x}=F(x)$. At jumps $(x \in D)$, the state value after the jump satisfies $x^{+}=G(x)$. The solution of a hybrid system is denoted $j, t \rightarrow x(t, j)$.
Then, (asymptotic) stability is defined as follows. A compact set $\mathcal{A}$ is stable for $\mathcal{H}$ if for each $\epsilon>0$ there exists $\sigma>0$ such that ${ }^{6}\|x(0,0)\|_{\mathcal{A}} \leq \sigma$ implies $\|x(t, j)\|_{\mathcal{A}} \leq \epsilon$ for all solutions $x$ to $\mathcal{H}$ and all $(t, j) \in \operatorname{dom} x$. A compact set is attractive if there exists a neighborhood of $\mathcal{A}$ from which each solution is complete and converges to $\mathcal{A}$, that is, $\|x(t, j)\|_{\mathcal{A}} \rightarrow 0$ as $t+j \rightarrow \infty$, where $(t, j) \in \operatorname{dom} x$. $\mathcal{A}$ is asymptotically stable if both properties hold.

In order to use the methods of Sanfelice et al. (2007) for the controlled rigid-body we define the error variable $\mathbf{e}_{h q}=$ $\left[1-h \tilde{\eta}, \tilde{\boldsymbol{\epsilon}}^{\top}\right]^{\top}$ which satisfies the kinematic equation

$$
\dot{\mathbf{e}}_{h q}=\mathbf{T}_{h}\left(\mathbf{e}_{h q}\right) \mathbf{e}_{\omega}
$$

where $\mathbf{e}_{\omega}=\boldsymbol{\omega}-\boldsymbol{\omega}_{d}$

$$
\mathbf{T}_{h}\left(\mathbf{e}_{h q}\right)=\left[\begin{array}{c}
h \tilde{\boldsymbol{\epsilon}}^{\top} \\
\tilde{\eta} \mathbf{I}+\mathbf{S}(\tilde{\boldsymbol{\epsilon}})
\end{array}\right]
$$

and $h \in H=\{-1,1\}$. The hybrid controller is composed of the continuous-time control law

$$
\begin{aligned}
\boldsymbol{\tau} & =\mathbf{J} \dot{\boldsymbol{\omega}}_{r}-\mathbf{S}(\mathbf{J} \boldsymbol{\omega}) \boldsymbol{\omega}_{r}-k_{q} \mathbf{T}_{h}^{\top} \mathbf{e}_{h q}-k_{\omega}\left(\boldsymbol{\omega}-\boldsymbol{\omega}_{r}\right), \\
\boldsymbol{\omega}_{r} & =\boldsymbol{\omega}_{d}-\gamma \mathbf{T}_{h}^{\top} \mathbf{e}_{h q}, \quad k_{q}, k_{\omega}, \gamma>0
\end{aligned}
$$

defining $\mathbf{x}=\left[\mathbf{e}_{h q}^{\top}, \mathbf{e}_{\omega}^{\top}, h\right]^{\top}$, the switching law

$$
\mathbf{x}^{+}=G(\mathbf{x})=\left[\mathbf{e}_{h q}^{\top}, \mathbf{e}_{\omega}^{\top},-h\right]^{\top} \mathbf{x} \in D
$$

and the flow and jump sets

$$
\begin{aligned}
& C=\left\{\mathbf{x} \in \mathcal{S}^{3} \times \mathbb{R}^{3} \times H: h\left[k_{q} \tilde{\eta}-\frac{1}{2} \gamma \tilde{\boldsymbol{\epsilon}}^{\top} \mathbf{J} \mathbf{e}_{\omega}\right] \geq-\delta\right\} \\
& D=\left\{\mathbf{x} \in \mathcal{S}^{3} \times \mathbb{R}^{3} \times H: h\left[k_{q} \tilde{\eta}-\frac{1}{2} \gamma \tilde{\boldsymbol{\epsilon}}^{\top} \mathbf{J} \mathbf{e}_{\omega}\right] \leq-\delta\right\},
\end{aligned}
$$

where $\delta$ defines the switching hysteresis, i.e., switching occurs only if the quantity involved in (20b) has decreased by certain amount. This is introduced to avoid Zeno behaviour.

Proposition 6 Consider the system (2) and (16)-(17) in closed loop with the controller (18)-(20). Then, the set

$$
\mathcal{A}=\left\{\mathbf{x} \in \mathcal{S}^{3} \times \mathbb{R}^{3} \times H:\left(\mathbf{e}_{h q}, \mathbf{e}_{\omega}\right)=(\mathbf{0}, \mathbf{0})\right\}
$$

is asymptotically stable on the set $\mathcal{S}^{3} \times \mathbb{R}^{3}$.

Proof: Let $s=\omega-\omega_{r}$ with $\omega_{r}$ defined by (18b). We use the Lyapunov function

$$
V_{s}(\mathbf{x})=\frac{1}{2}\left(\mathbf{s}^{\top} \mathbf{J} \mathbf{s}+\mathbf{e}_{h q}^{\top} k_{q} \mathbf{e}_{h q}\right)
$$

whose total time derivative along the closed-loop flow trajectories, that is generated by (2), (16)-(17) and (18), yields (under the quaternion constraint)

$$
\dot{V}_{s}(\mathbf{x}) \leq-\mathbf{s}^{\top} k_{\omega} \mathbf{s}-\mathbf{e}_{h q}^{\top} \frac{\gamma k_{q}}{8} \mathbf{e}_{h q}
$$

$\overline{{ }^{6} \text { As usual, }}\|x\|_{\mathcal{A}}=\inf _{z \in \mathcal{A}}|z-x|$. 
-see (9). Now, we evaluate $V_{s}(G(\mathbf{x}))-V_{s}(\mathbf{x})$. We have

$$
\begin{gathered}
V_{s}(G(\mathbf{x}))=\frac{1}{2}\left[\left(\mathbf{e}_{\omega}+\gamma \mathbf{T}_{-h}^{\top} \mathbf{e}_{h q}\right)^{\top} \mathbf{J}\left(\mathbf{e}_{\omega}+\gamma \mathbf{T}_{-h}^{\top} \mathbf{e}_{h q}\right)\right. \\
\left.+\mathbf{e}_{h q}^{\top} k_{q} \mathbf{e}_{h q}\right] \\
V_{s}(\mathbf{x})=\frac{1}{2}\left[\left(\mathbf{e}_{\omega}+\gamma \mathbf{T}_{h}^{\top} \mathbf{e}_{h q}\right)^{\top} \mathbf{J}\left(\mathbf{e}_{\omega}+\gamma \mathbf{T}_{h}^{\top} \mathbf{e}_{h q}\right)+\mathbf{e}_{h q}^{\top} k_{q} \mathbf{e}_{h q}\right]
\end{gathered}
$$

Note that $\mathbf{T}_{h}^{\top} \mathbf{e}_{h q}=1 / 2 h \tilde{\boldsymbol{\epsilon}}$ and that $\mathbf{e}_{h q}^{\top} \mathbf{e}_{h q}=(1-h \tilde{\eta})^{2}+$ $\tilde{\boldsymbol{\epsilon}}^{\top} \tilde{\boldsymbol{\epsilon}}$, and by inserting the unit quaternion constraint $\tilde{\eta}^{2}+$ $\tilde{\boldsymbol{\epsilon}}^{\top} \tilde{\boldsymbol{\epsilon}}=1$ we have $\mathbf{e}_{h q}^{\top} \mathbf{e}_{h q}=2(1-h \tilde{\eta})$. Hence,

$$
\begin{array}{r}
2 V_{s}(G(\mathbf{x}))=\mathbf{e}_{\omega}^{\top} \mathbf{J} \mathbf{e}_{\omega}-\gamma(-h) \tilde{\boldsymbol{\epsilon}}^{\top} \mathbf{J} \mathbf{e}_{\omega}+\frac{1}{4} \gamma^{2}(-h)^{2} \tilde{\boldsymbol{\epsilon}}^{\top} \mathbf{J} \tilde{\boldsymbol{\epsilon}} \\
+2 k_{q}[1-(-h) \tilde{\eta}] \\
2 V_{s}(\mathbf{x})=\mathbf{e}_{\omega}^{\top} \mathbf{J} \mathbf{e}_{\omega}-\gamma h \tilde{\boldsymbol{\epsilon}}^{\top} \mathbf{J} \mathbf{e}_{\omega}+\frac{1}{4} \gamma^{2} h^{2} \tilde{\boldsymbol{\epsilon}}^{\top} \mathbf{J} \tilde{\boldsymbol{\epsilon}}+2 k_{q}[1-h \tilde{\eta}]
\end{array}
$$

and therefore

$$
V_{s}(G(\mathbf{x}))-V_{s}(\mathbf{x})=2 h\left[k_{q} \tilde{\eta}-\frac{1}{2} \gamma \tilde{\boldsymbol{\epsilon}}^{\top} \mathbf{J} \mathbf{e}_{\omega}\right]
$$

which in view of (20b), implies $V_{s}(G(\mathbf{x}))-V_{s}(\mathbf{x}) \leq-2 \delta<0$ when $\mathbf{x} \in D$. Thus,

$$
\begin{array}{cl}
\dot{V}_{s}(\mathbf{x}) \leq-c_{3} / c_{2} V_{s}(\mathbf{x}) & \forall \mathbf{x} \in C \\
V_{s}(G(\mathbf{x}))-V_{s}(\mathbf{x})<0 & \forall \mathbf{x} \in D .
\end{array}
$$

After (Sanfelice et al., 2007, Corollary 7.7) we conclude that the set $\mathcal{A}$ is asymptotically stable with basin of attraction $\mathcal{B}_{\mathcal{A}}=C \cup D$, thus uniform asymptotic stability in the large on the set $\mathcal{S}^{3} \times \mathbb{R}^{3}$ follows.

\section{Comparative simulations study}

We have performed several series of simulations to compare the hybrid controller of Section 3.3 to the continuous controller of Proposition 5. In the simulations setting we assume that the rigid body is a spacecraft and naturally, the primary goal is to improve performance. The latter is measured in terms of (reducing) fuel consumption, i.e., control effort. For that purpose we use the functionals

$$
J_{q}=\int_{t_{0}}^{t_{f}} \tilde{\boldsymbol{\epsilon}}^{\top} \tilde{\boldsymbol{\epsilon}} d t, \quad J_{\omega}=\int_{t_{0}}^{t_{f}} \boldsymbol{e}_{\omega}^{\top} \boldsymbol{e}_{\omega} d t, \quad J_{p}=\int_{t_{0}}^{t_{f}} \boldsymbol{\tau}_{a}^{\top} \boldsymbol{\tau}_{a} d t
$$

where $t_{0}$ and $t_{f}$ define the start and end of the simulations window respectively. The functionals $J_{q}$ and $J_{\omega}$ correspond to the integral square errors of the attitude and the angular velocity error respectively. The functional $J_{p}$ is a measure of the energy injected to perform the attitude maneuver. Thus, the controllers of the previous sections are implemented with similar tuning but with the aim at reducing $J_{p}$.

The simulations are performed in Simulink ${ }^{\mathrm{TM}}$ using a fixed sample-time Runge-Kutta ODE4 solver, with sample time of $1 \cdot 10^{-2} \mathrm{~s}$. The moments of inertia for the rigid body are $\mathbf{J}=\operatorname{diag}\{4.35,4.33,3.664\} \mathrm{kgm}^{2}$.

For the continuous controller of Proposition 5 a reference equilibrium point is chosen a priori according to the method
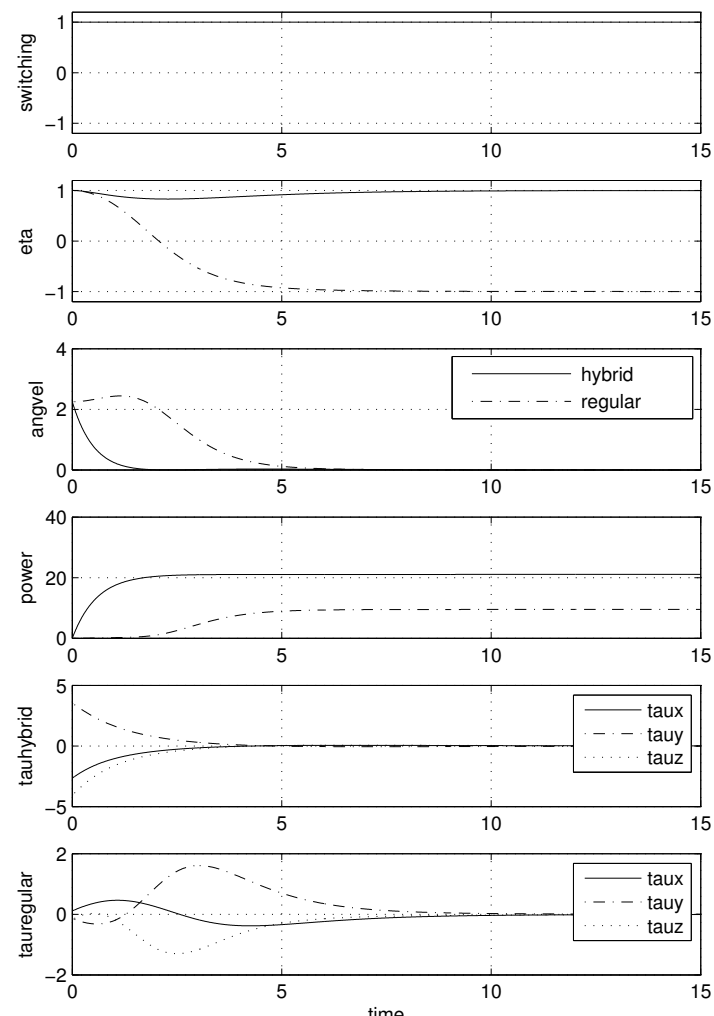

Fig. 1. Switching value, attitude error, total angular velocity error, power consumption and actuator torque for switching and continuous sliding surface control of a rigid body. $\left(k_{q}=1, k_{\omega}=2, \boldsymbol{\omega}=1.5 \mathrm{v}\right)$

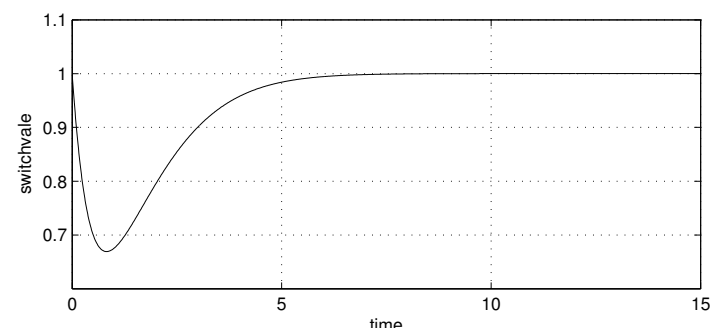

Fig. 2. Switching criterion evolving over time

described in Schlanbusch et al. (2010b). This is done considering both initial sense of rotation and orientation, i.e.,

$$
\mathbf{e}_{q}=\left\{\begin{array}{l}
\mathbf{e}_{q+} \text { if } k_{\tilde{\eta}} \tilde{\eta}\left(t_{0}\right)+k_{\dot{\eta}} \dot{\tilde{\eta}}\left(t_{0}\right) \geq 0 \\
\mathbf{e}_{q-} \text { if } k_{\tilde{\eta}} \tilde{\eta}\left(t_{0}\right)+k_{\dot{\tilde{\eta}}} \dot{\tilde{\eta}}\left(t_{0}\right)<0
\end{array},\right.
$$

where $\tilde{\eta}\left(t_{0}\right)$ and $\dot{\tilde{\eta}}\left(t_{0}\right)$ are initial values and $k_{\tilde{\eta}}$ and $k_{\dot{\tilde{\eta}}}$ are design parameters. Note that $\dot{\tilde{\eta}}=-1 / 2 \tilde{\boldsymbol{\epsilon}}^{\top} \mathbf{e}_{\omega}$. By this rule of choice we can make a fair comparison with the hybrid controller.

Also, an appropriate tuning of the control gains is made. For the continuous controller the gains $k_{\tilde{\eta}}$ and $k_{\dot{\tilde{\eta}}}$ can be tuned to fit a given system while for the switching strategy tuning consists in choosing appropriately the hysteresis threshold $\delta$ and the gains $k_{q}$ and $\gamma$ which reduces the tuning flexibility 

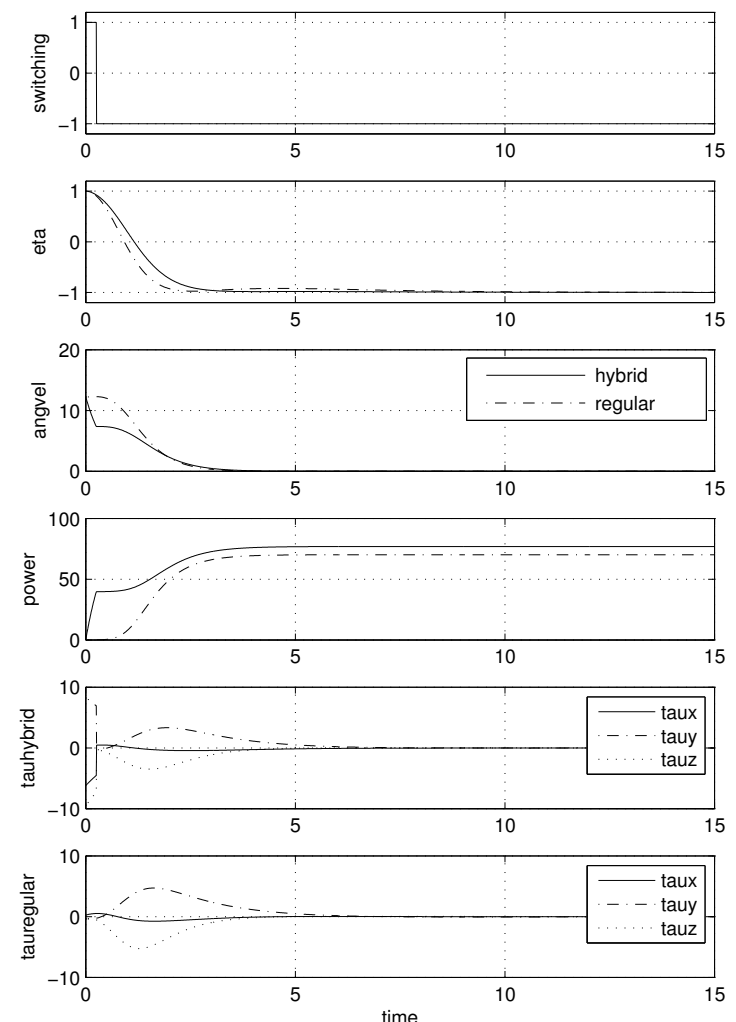

Fig. 3. Switching value, attitude error, total angular velocity error, power consumption and actuator torque for switching and continuous sliding surface control of a rigid body. $\left(k_{q}=1, k_{\omega}=2, \boldsymbol{\omega}=3.5 \mathbf{v}\right)$

of the control law itself.

An interesting natural scenario to consider is when the initial conditions are 'large'. Particularly, when the spacecraft's attitude is 'close' to the desired one but turning rapidly away from it, that is, $\left\|\boldsymbol{e}_{\omega}\left(t_{\circ}\right)\right\| \gg 1$ and $\tilde{\boldsymbol{\epsilon}} \approx 0$. Note that the switching law in (20) consists in verifying whether

$$
\sigma:=h\left(k_{q} \tilde{\eta}-\frac{1}{2} \gamma \tilde{\boldsymbol{\epsilon}}^{\top} \mathbf{J} \mathbf{e}_{\omega}\right) \geq-\delta
$$

Hence, if we have $\tilde{\boldsymbol{\epsilon}}^{\top} \mathbf{e}_{\omega} \approx 0$ the effect of (large) angular velocity errors may be transparent to the hybrid controller. The simulations are made for two cases of initial angular velocities. For the plots showed in Figure 1 we used $\mathbf{q}\left(t_{\circ}\right)=[1, \mathbf{0}]^{\top}$ and $\boldsymbol{\omega}\left(t_{\circ}\right)=1.5 \mathbf{v}^{\top}$ where $\mathbf{v}=$ $[3,-4,5]^{\top} /\left\|[3,-4,5]^{\top}\right\|$, and the gains are set to $k_{q}=1$, $k_{\omega}=2, \gamma=1$, with switching threshold $\delta=0.1$. As it may be appreciated the negative equilibrium point is preferable but the switching condition for the hybrid controller is never met; the trajectories remain out of the jump set. One may think that the hysteresis switching surface is not chosen properly however, Figure 2 illustrates the opposite. It shows that the value of the switching function does not reach the jump condition for any $\delta>0$. It may be argued that the initial angular velocity may not be recognized by the continuous controller (21) either. Yet, this expression
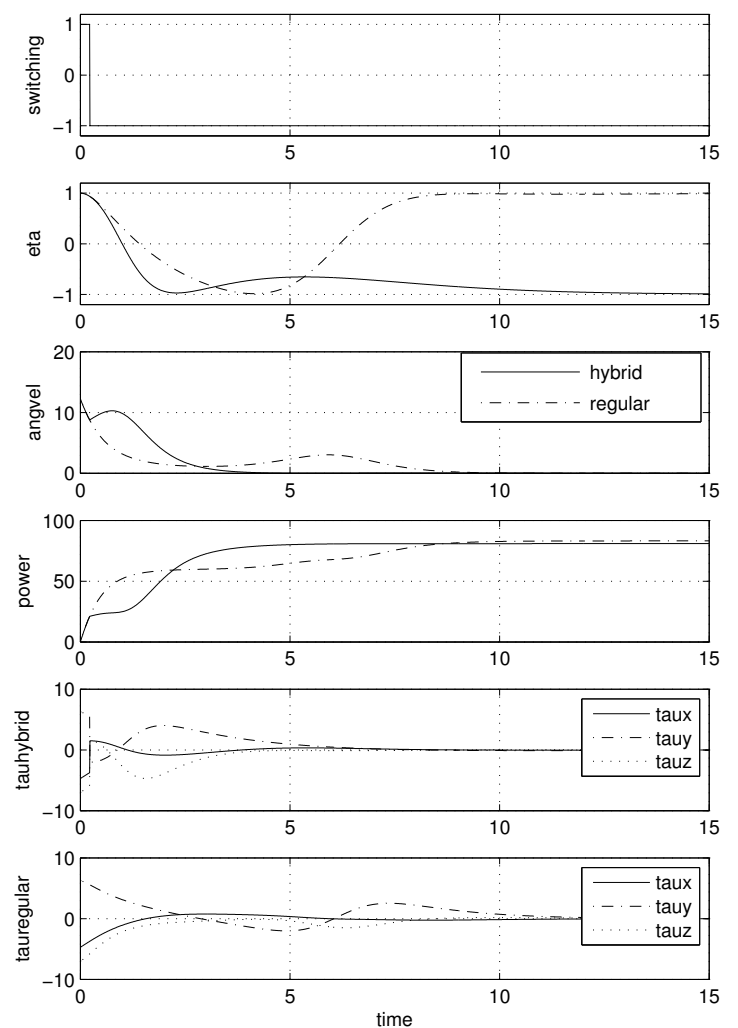

Fig. 4. Switching value, attitude error, total angular velocity error, power consumption and actuator torque for switching and continuous sliding surface control of a rigid body. $\left(k_{q}=1, k_{\omega}=1, \boldsymbol{\omega}=3.5 \mathbf{v}\right)$

only accounts for small initial angular velocities. For large initial angular velocities the equilibrium point giving the largest initial rotational error is in general preferred.

Next, we increase the initial velocity to $\boldsymbol{\omega}\left(t_{\circ}\right)=3.5 \mathbf{v}^{\top}$ to provoke switching; see Figure 3 . Now, the system under either controller, continuous or hybrid, eventually settles at the negative equilibrium point, although, the continuous controller is faster, consumes less energy and utilize less maximum torque but leads to larger overshoot of the attitude error.

To compare further the performances of the hybrid versus the continuous controller we performed simulations by decreasing the derivative gain to $k_{\omega}=1$. On one hand, this entails a change in the preferred reference equilibrium point when using the continuous controller $(5)^{7}$. On the other, using the hybrid controller with lower derivative gain value results in lower energy consumption and smaller overshoot of the attitude absolute error. See Figure 4. The energy consumption under hybrid control may be further diminished by increasing the hysteresis threshold $\delta$.

A comparison of the controllers in function of the perfor-

7 It should be noted that according to Schlanbusch et al. $(2010 a)$ it is assumed that the available actuator torque is sufficient and that the control law is tuned to make the rigid body stop at the preferred equilibrium point without doing any full revolutions, thus the system in this case is under-damped. 
mance indexes $J_{p}, J_{\omega}$ and $J_{\tau}$ is presented in Table 1 .

Table 1

Values of performance functionals

\begin{tabular}{|l|l|l|l|}
\hline & $J_{q}$ & $J_{\omega}$ & $J_{p}$ \\
\hline Continuous controller (Fig. 1) & 2.80 & 7.19 & 9.50 \\
\hline Hybrid controller (Fig. 1) & 1.47 & 1.15 & 21.07 \\
\hline Continuous controller (Fig. 3) & 1.86 & 18.00 & 70.11 \\
\hline Hybrid controller (Fig. 3) & 1.69 & 13.44 & 76.89 \\
\hline Continuous controller (Fig. 4) & 4.52 & 20.02 & 83.16 \\
\hline Hybrid controller (Fig. 4) & 4.53 & 19.44 & 80.95 \\
\hline
\end{tabular}

\section{Conclusions}

We analyzed the control attitude problem under three different settings. Although the basic control law is the same, the difference lies in the theoretical assumptions that are made. The specificities of each setting requires the use of especial stability tools, each leading to a different mathematical result. From a practical viewpoint, it is obtained for the three cases that the attitude errors converge to zero from almost any initial valid posture. However, it has been showed in simulations that performance may be improved if, for instance, it is allowed to change the reference equilibrium point during the maneuver.

Although this is not discussed due to space constraints, the results here presented may be extended to the more realistic case in which a spacecraft is affected by unknown bounded matched disturbances.

\section{Acknowledgements}

The authors express their gratitude to Editor Teel and the Associate Editor for the exceptional handling of this paper and greatly appreciate the many constructive remarks of the anonymous reviewers.

\section{References}

Angeli, D. (2001). Almost global stabilization of the inverted pendulum via continuous state feedback. Automatica 37(7), 1103-1108.

Angeli, D. (2004). An almost global notion of input-to-state stability. IEEE Transactions on Automatic Control 49(6), 866874.

Caccavale, F. and L. Villani (1999). Output feedback control for attitude tracking. Systems \& Control Letters 38(2), 91-98.

Casagrande, D., A. Astolfi and T. Parisini (2008). Global asymptotic stabilization of the attitude and the angular rates of an underactuated non-symmetric rigid body. Automatica 44(7), 1781-1789.

Egeland, O. and J. T. Gravdahl (2002). Modeling and Simulation for Automatic Control. Marine Cybernetics. Trondheim, Norway. ISBN 82-92356-01-0.

Furasov, V. D. (1977). Ustoichivost' dvijeniya, otzenki i stabilizatsia. Nauka. Moscow. Translated title: Stability of motion, estimates and stabilization.

Goebel, R., R. G. Sanfelice and A. R. Teel (2009). Hybrid dynamical systems. IEEE Control Systems Magazine 29(2), 2893.

Hahn, W. (1967). Stability of Motion. Springer-Verlag. Berlin, Germany.

Kristiansen, R., P. J. Nicklasson and J. T. Gravdahl (2008). Spacecraft coordination control in 6DOF: Integrator backstepping vs passivity-based PD+. Automatica 44(11), 28962901.
Loría, A. and E. Panteley (2006). Stability, told by its developers. In: Advanced Topics in Control Theory (A. Loría, F. Lamnabhi-Lagarrigue and E. Panteley, Eds.). Vol. 328 of Lecture Notes in Control and Information Sciences. Chap. 6, pp. 199-258. Springer Verlag. London. ISBN 1-84628-313-2.

Mayhew, C. G., R. G. Sanfelice and A. R. Teel (2009). Robust global asymptotic attitude stabilization of a rigid body by quaternion-based hybrid feedback. In: Proceedings of the IEEE Conference on Decision and Control.

Monzón, P. (2006). Almost global stability of time-varying systems. In: Proceedings of the Congresso Brasileiro de Automatica. Bahia, Brasil. pp. 198-201.

Rantzer, A. (2001). A dual to Lyapunov's stability theorem. Systems \& Control Letters 42(3), 161-168.

Sanfelice, R. G., R. Goebel and A. R. Teel (2007). Invariance principles for hybrid systems with connections to detectability and asymptotic stability. IEEE Transactions on Automatic Control 52(12), 2282-2297.

Schlanbusch, R., R. Kristiansen and P. J. Nicklasson (2010a). Considerations choosing the optimal equilibrium point on the rotational sphere. In: Proceedings of the American Control Conference. Baltimore, MD.

Schlanbusch, R., R. Kristiansen and P. J. Nicklasson (2010b). On choosing quaternion equilibrium point in attitude stabilization. In: Proceedings of the 31th IEEE Aerospace Conference. Big Sky, MO.

Slotine, J. J.-E. and W. Li (1988). Adaptive manipulator control: A case study. Automatic Control, IEEE Transactions on 33(11), 995-1003.

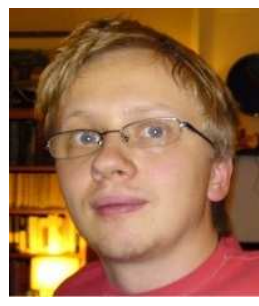

Rune Schlanbusch received his BSc, MSc and $\mathrm{PhD}$ degrees in Space technology from Narvik University College, Norway, in 2005, 2007 and 2012, respectively, and currently holds a position as associate professor at the same institute. His research interests include rotational and translational control of rigid bodies with special focus on spacecraft and spacecraft formations.

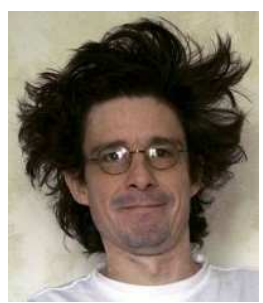

Antonio Loría got the BSc degree in Electronic Engineering from the ITESM, Monterrey, Mexico in 1991, the MSc and PhD degrees in Control Engg. from the UTC, France in 1993 and 1996 respectively. He occupied post-doctoral positions at Univ. of Twente, The Netherlands (1996-1997); NTNU, Norway (1997) and Univ. of California at Sta Barbara, USA (1998). A. Loria has the honour of holding a research position as "Directeur de Recherche" (senior researcher), at CNRS, France. His research interests include control systems theory and practice, electrical systems, analysis and control of chaos. He serves as associate editor for Systems and Control Letters, Automatica, IEEE Transactions on Automatic Control, IEEE Transactions on Control Systems Technology and is memeber of the IEEE CSS Conference Editorial Board. See also http://www.l2s.supelec.fr/perso/loria.

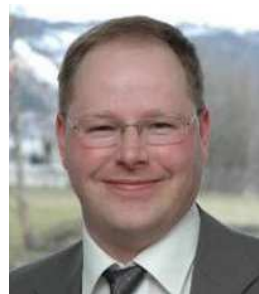

Per Johan Nicklasson received the M.Sc. and the Ph.D. degrees in engineering cybernetics from NTH, Trondheim, Norway, in 1991 and 1996 respectively. He is currently professor in electrical engineering at Narvik University College, Norway. His fields of interest are nonlinear control and cold climate technology. 